\begin{tabular}{|c|c|}
\hline Title & Topology-change surgery for crystals \\
\hline Author(s) & Matsuura, T.; Tsuneta, T.; Kumagai, G.; T subota, M.; Matsuyama, T.; Tanda, S. \\
\hline Citation & $\begin{array}{l}\text { Physical Review B, 83(17), } 174113 \\
\text { https://doi.org/10.1103/PhysRevB.83.174113 }\end{array}$ \\
\hline Issue Date & 2011-05-01 \\
\hline Doc URL & http:/hdl.handle.net/2115/45808 \\
\hline Rights & @2011 A merican Physical Society \\
\hline Type & article \\
\hline File Information & PRB83-17_174113.pdf \\
\hline
\end{tabular}

Instructions for use 


\title{
Topology-change surgery for crystals
}

\author{
T. Matsuura, ${ }^{1}$ T. Tsuneta, ${ }^{2}$ G. Kumagai, ${ }^{3}$ M. Tsubota, ${ }^{3}$ T. Matsuyama,${ }^{2}$ and S. Tanda ${ }^{3,4}$ \\ ${ }^{1}$ Research Department, Creative Research Institute, Hokkaido University, Sapporo, Hokkaido 001-0021, Japan \\ ${ }^{2}$ Graduate School of Education, Nara University of Education, Nara 630-8528, Japan \\ ${ }^{3}$ Department of Applied Physics, Hokkaido University, Sapporo, Hokkaido 060-8628, Japan \\ ${ }^{4}$ Center of Education and Research for Topological Science and Technology, Hokkaido University, Sapporo, Hokkaido 060-8628, Japan
}

(Received 3 March 2011; published 31 May 2011)

\begin{abstract}
We performed the topology-change surgery for ring-shaped crystals of tantalum triselenide $\left(\mathrm{TaSe}_{3}\right)$ to investigate the interplay between the closed-ring topology and elasticity/plasticity of the crystals. We cut the $\mathrm{TaSe}_{3}$ rings using a focused ion beam and observed that the curvatures of the open rings increased from their initial curvatures. We found that a change in the radius is proportional to inverse square of the thickness of the crystals, which corresponds to an inhomogeneous distribution of edge dislocations. From the distribution, we suggest the existence of cylindrical domain walls in the ring-shaped crystals as a result of the crystal topology.
\end{abstract}

DOI: 10.1103/PhysRevB.83.174113

PACS number(s): 02.40.Pc, 61.72.Hh, 61.72.Lk

The surgery method in Mathematics, which changes the topology of a space by cut and glue, is a powerful tool to extract topological properties, as Perelman solved the Poincaré conjecture. ${ }^{1,2}$ Even in nature, the topology-change surgery is an important approach since the interplay between global topology and local symmetry would play a fundamental role in cosmology, condensed matter physics, and biology. ${ }^{3-5}$ By the discovery of rings, Möbius rings and Hopf links of crystals of transition metal-trichalcogenide compound systems $\left(\mathrm{NbSe}_{3}\right.$, $\mathrm{NbS}_{3}, \mathrm{TaSe}_{3}$, and $\mathrm{TaS}_{3}$ ), ${ }^{6-9}$ we obtained experimental systems for the interplay between the closed-ring topology and local order parameters. Using the topological crystals, effects of the closed-ring topology on charge density waves ${ }^{10-17}$ and superconductivity ${ }^{18}$ have been investigated.

As well as the ordered phases in electron systems, the crystal orders of the topological crystals have rich problems of the interplay between the global topology. The crystal structures of the topological crystals must be different from normal crystals due to a topological charge, namely, a global disclination. ${ }^{19}$ As shown in Fig. 1(a) (left), the global disclination is obtained as the total rotation angle of a crystal axis (b axis) of a ring-shaped crystal along a closed curve on the crystal, where it is $2 \pi$ for the ring-shaped crystals, and this value is topologically robust against continuous deformations. Due to the global disclination of power $2 \pi$, the crystal lattice cannot be mapped onto that of perfect crystals globally. Moreover, the topological crystals must be frustrated geometrically by the deformation energy from bending or twist, and the deformation energy would induce topological defects into a topological crystal for relaxation. Hayashi et al. pointed out theoretically that the geometrical frustrations (curvature) of the crystals induce topological defects (edge dislocations), as analogous with vortices in type II superconductors. ${ }^{20}$ The $\mathrm{x}$-ray diffraction measurements for ring-shaped crystals of $\mathrm{NbSe}_{3}$ confirmed that the averaged bending strain in a ring-shaped crystal is relaxed by defects. ${ }^{21}$ However, the distributions of strain and defects have never investigated experimentally since the $\mathrm{x}$-ray diffraction measurement has less spatial resolution for the small crystals due to the spot size of $x$ ray. In principle, the conventional methods with the plane waves of x-ray/electronbeam diffraction are inappropriate for investigation of the curved crystals since the directions of the crystal lattices are depend on the position.

In this paper, we performed the topology-change surgery experiment with the ring-shaped crystals of tantalum triselenide $\left(\mathrm{TaSe}_{3}\right)$ to reveal the interplay between the global topology and the local crystal properties (elasticity/plasticity). The crystal structure of $\mathrm{TaSe}_{3}$ is orthorhombic with lattice constants $a=10.402 \AA, b=3.495 \AA, c=9.829 \AA$, and $\beta=106.26^{\circ},{ }^{22}$ and $\mathrm{TaSe}_{3}$ crystals are known to be superconductors. ${ }^{23}$ The $\mathbf{b}$ axis is parallel to the circumference of the ring-shaped crystals similar to the ring-shaped crystals of $\mathrm{NbSe}_{3} .6,7,24$ When a ring-shaped crystal is cut as shown in Fig. 1(a) (right), the global disclination becomes zero. Thus, the topology-change surgery from closed-ring to open-ring crystals means eliminating the global disclination of power $2 \pi$ from the crystals similar to that the vortex in a superconductor ring is eliminated when the ring was cut. As the results, we discovered that the changes of the curvatures are proportional to inverse square of the thickness, which corresponds to the nonlinearity of the defect creation. The analysis based on the elastic theory suggests that the line defects (edge dislocations) form a "topological domain wall."

To cut the ring-shaped crystals, we used the irradiation of a focused gallium ion beam with a spot size of less than $1 \mu \mathrm{m}$ (acceleration voltage: $30 \mathrm{kV}) \cdot{ }^{14,25}$ Figures $1(\mathrm{~b})$ and 1 (c) show the scanning ion beam microscopy (SIM) images of the topology-change surgery experiment for typical thin and thick ring-shaped crystals, respectively, where each left panel shows the original shapes of the ring-shaped crystals and each right panel shows the final shapes after the topologychange surgery. We observed that the thin ring-shaped crystals opened elastically after the surgery; however, no open-ring crystals became to be straight (zero curvature), while the global disclination became zero. These results indicate that the bending stress is partially relaxed by the defects.

To evaluate the elasticity of the crystals, we introduced the dimensionless parameter $\left(R_{\mathrm{f}}-R_{0}\right) / R_{0}$, where we defined the initial and the final radiuses of curvatures $\left(R_{0}\right.$ and $\left.R_{\mathrm{f}}\right)$ as average values for the outer and inner radiuses, and thickness $T$ as differential of the outer and inner radiuses. Note that the shapes of the open-ring crystals could be fitted by circular 
(a)
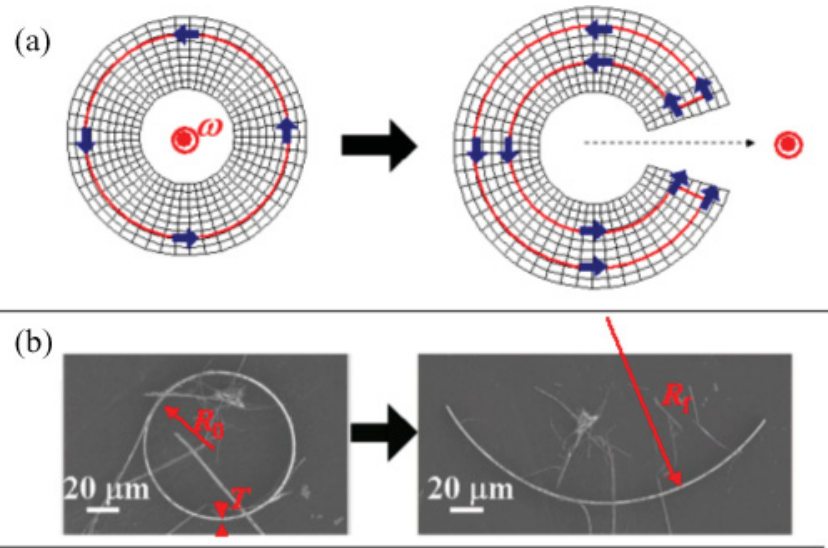

(c)
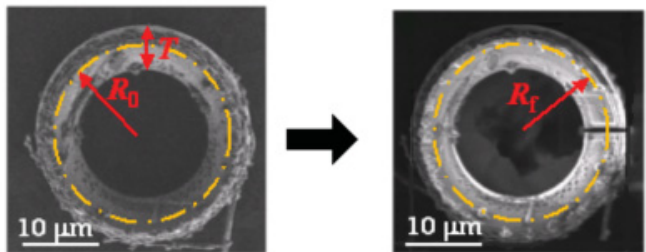

(d)

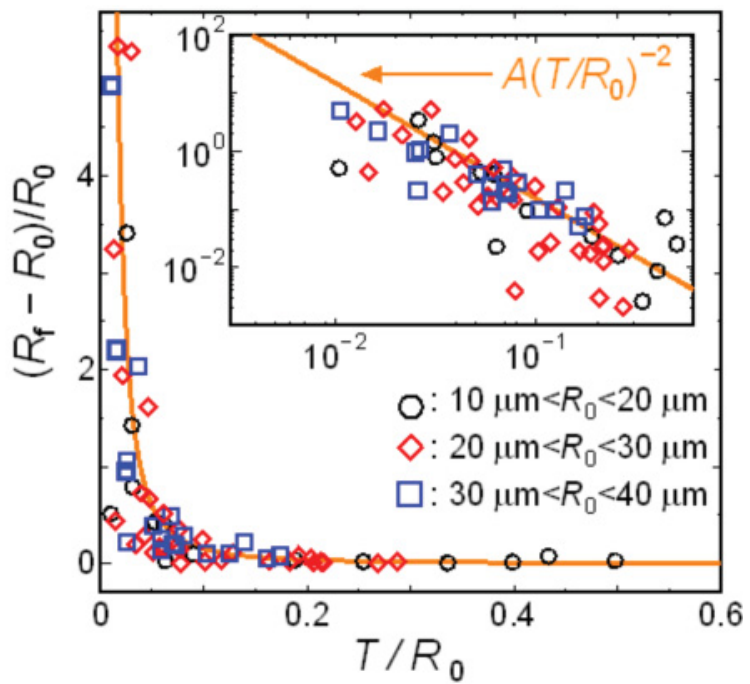

FIG. 1. (Color online) (a) Schematics of topology-change surgery for a ring-shaped crystal. The global disclination of power $\omega=\oint_{C} d \theta_{\mathbf{b}}$ is changed by surgery from $2 \pi$ (for the closed-ring crystal in the left panel) to zero (for the open-ring crystal in the right), where $\theta_{\mathbf{b}}$ is relative angle of $\mathbf{b}$ axis of $\mathrm{TaSe}_{3}$ crystals (indicated by the arrows). (b) and (c) Scanning ion beam microscopy (SIM) images of typical thin and thick ring-shaped crystals of $\mathrm{TaSe}_{3}$ before topology-change surgery (left), and after surgery (right). The thickness $T$, original radius of ring-shaped crystals $R_{0}$, and final radius of open-ring crystals after topology change $R_{\mathrm{f}}$ are $1.583,42.45$, and $128.4 \mu \mathrm{m}$ for the thin ring-shaped crystal (b) and $6.00,12.05$, and $12.35 \mu \mathrm{m}$ for the thick ring-shaped crystal (c), respectively. (d) Ratio of curvatures $\left(R_{\mathrm{f}}-R_{0}\right) / R_{0}$ as a function of normalized thickness $T / R_{0}$. The log-log plot is shown in the inset. The solid line indicates the fitting curve given by $A\left(T / R_{0}\right)^{\alpha}$, where we use $\alpha=-2$ and $A=0.0015$.

arcs, elliptic arcs, or cycloid/trochoid curves; however, we consider all of them to be circular arcs with a mean radius of curvature $R_{\mathrm{f}}$ as the zeroth approximation in this paper. If a ring-shaped crystal were made from a perfect crystal (without defects), $R_{\mathrm{f}}$ should be infinity, since all bending stress could be elastically relaxed by the topology-change surgery from closed ring to open ring. On the other hand, if the bending stress in the ring-shaped crystal were completely plastically relaxed by defects, the curvature would not be changed by the topology change; in this case, $\left(R_{\mathrm{f}}-R_{0}\right) / R_{0}=0$. Hence, the value of $\left(R_{\mathrm{f}}-R_{0}\right) / R_{0}$ represents the number of the defects inside the ring-shaped crystal.

Figure 1(d) shows the experimental data of $\left(R_{\mathrm{f}}-R_{0}\right) / R_{0}$ as a function of the normalized thickness $T / R_{0}$. The colors of the symbols correspond to the ranges of the initial radius $R_{0}$, and we found that $\left(R_{\mathrm{f}}-R_{0}\right) / R_{0}$ did not depend on $R_{0}$. The ring-shaped crystals with small $T / R_{0}$ values $\left(T / R_{0}<0.05\right)$ were elastically opened by the topology-change surgery, and for some ring-shaped crystals, the final radiuses of curvatures $R_{\mathrm{f}}$ were more than double $R_{0}$, and all the crystals were in the $\left(R_{\mathrm{f}}-R_{0}\right) / R_{0}<6$ range. We note that the distribution of $\left(R_{\mathrm{f}}-R_{0}\right) / R_{0}$ did not depend on the width of the crystal. Thus, we will not discuss the width. From the log-log plot of $\left(R_{\mathrm{f}}-R_{0}\right) / R_{0}$ versus $T / R_{0}$ shown in the inset of Fig. $1(\mathrm{~d})$, we found the following relation:

$$
R_{\mathrm{f}}=R_{0}\left(1+A\left(\frac{T}{R_{0}}\right)^{\alpha}\right)
$$

A fitting line with $\alpha=-2$, and $A=0.0015$ is plotted in Fig. 1(d) and in its inset. The second term of Eq. (1) indicates the plasticity of the ring-shaped crystals. As discussed later, $A$ and $\alpha$ involve information about the yield strain and strain distribution, respectively, reflecting the anisotropic crystal structure of $\mathrm{TaSe}_{3}$.

We investigated Eq. (1) using a model of ring-shaped crystals including defects based on the elastic theory. ${ }^{26}$ Here, we here assumed that $R_{\mathrm{f}} / R_{0}$ depends solely on strain for the b axis (parallel to the circumference), while the stress and strain in the orthorhombic system of $\mathrm{TaSe}_{3}$ crystals should be strictly expressed by tensors. This assumption is applicable to the ring-shaped crystals since recent results from the $\mathrm{x}$-ray diffraction measurement for the ring-shaped crystals of $\mathrm{NbSe}_{3}$ provided corroboration that the strain along the $\mathbf{b}$ axis is much larger than those for other axes. ${ }^{21}$ Hence, in this paper, we considered only the strain along the $\mathbf{b}$ axis. If there are edge dislocations in the bent material, the free length of the circumference (parallel to $\mathbf{b}$ ) is written as $2 \pi R_{0}\left(1-\epsilon_{\mathrm{d}}(x)\right.$ ), where $\epsilon_{\mathrm{d}}(x)$ represents the change in the lattice number along the circumference caused by the edge dislocations. The strain with an arbitrary radius of curvature $R$ is expressed as

$$
\epsilon_{\mathrm{r}}(x, R) \sim \frac{x}{R}+\epsilon_{\mathrm{d}}(x),
$$

where we assumed $\epsilon_{\mathrm{d}}(x) \ll 1$. Note that, if the density of dislocations is a constant value $\left[\epsilon_{\mathrm{d}}(x)=-\gamma x / R_{0}(1 \geqslant \gamma \geqslant\right.$ $0)], \epsilon_{\mathrm{r}}(x, R)$ is constant, and then $\alpha$ in Eq. (1) is 0 . The bending energy density is written as $f(x, R)=\frac{Y}{2} \epsilon_{\mathrm{r}}(x, R)^{2}$, where the 

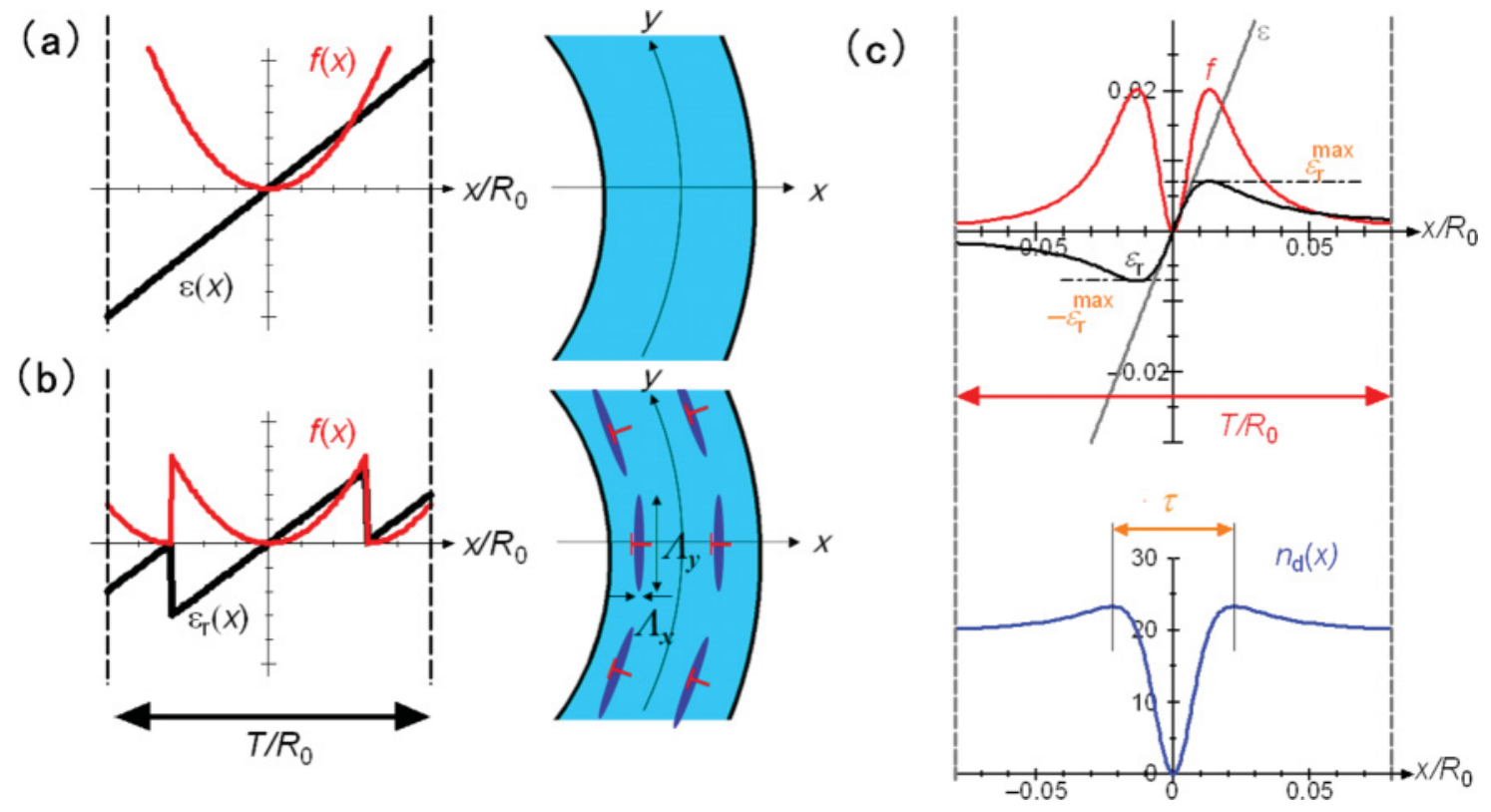

FIG. 2. (Color online) (a) Schematics of strain $\epsilon_{\mathrm{r}}(x, R)$ and bending energy $f(x, R) \propto \epsilon_{\mathrm{r}}(x, R)^{2}$ for a bent perfect crystal. The bending strain $\epsilon$ with a radius of curvature $R$ is expressed as $\epsilon=\frac{x}{R}$, where $x$ is the distance from a center neutral axis along the radial direction. The bending stress is assumed to be proportional to strain $\epsilon$, such as $\sigma=Y \epsilon$, where $Y$ is Young's modulus. The free energy density of the bending strain is expressed as $f(x, R)=\int \sigma(\epsilon) d \epsilon$, which is shown as a red curve. (b) Model of a bent crystal with edge dislocations. The strain $\epsilon_{r}$ is relaxed by the edge dislocations. Because of the large anisotropy of the crystal, the core sizes of the edge dislocations are strongly elongated to $\mathbf{b}$ axis direction, where $\Lambda_{y}$ is about 10 times larger than $\Lambda_{x}$. (c) Strain $\epsilon_{\mathrm{r}}\left(x, R_{0}\right)$ [Eq. (5) with $A=0.0015$ ], bending energy $f\left(x, R_{0}\right)$, and density of edge dislocations $n_{\mathrm{d}}(x)$. The bottom panel shows $n_{\mathrm{d}}(x)$ calculated for $R_{0}=30 \mu \mathrm{m}$. $n_{\mathrm{d}}(x)$ reaches its maximum value at $|x| / R_{0}=\tau / 2 \equiv \sqrt{\left(\sqrt{\left.\frac{5}{2}-\frac{5}{4}\right) A}\right.} \sim 0.575 \sqrt{A}$, and then decreases to a constant value.

stress is assumed as $\sigma_{r}(x, R)=Y \epsilon_{r}(x, R)$. The total elastic energy of the open-ring crystal is

$$
\begin{aligned}
F(R) & =\int_{-W / 2}^{W / 2} \int_{0}^{2 \pi R_{0}\left(1-\epsilon_{\mathrm{d}}(x)\right)} \int_{-T / 2}^{T / 2} f(x, R) d x d y d z \\
& =\pi Y R_{0} W \int_{-T / 2}^{T / 2} \epsilon_{\mathrm{r}}(x, R)^{2}\left(1-\epsilon_{\mathrm{d}}(x)\right) d x
\end{aligned}
$$

Thus, we obtained the condition of the final radius of curvature of the open-ring crystals by $\left.\frac{d F(R)}{d R}\right|_{R=R_{\mathrm{f}}}=0$, as follows:

$$
\frac{1}{R_{\mathrm{f}}}=-\frac{12}{T^{3}} \int_{-T / 2}^{T / 2} x \epsilon_{\mathrm{d}}(x) d x .
$$

Here, we note that $R_{\mathrm{f}}$ does not depend on $Y . \epsilon_{\mathrm{d}}(x)$ in the ring-shaped crystals is obtained from Eq. (4) by using Eq. (1) with $\alpha=-2$ :

$$
\epsilon_{\mathrm{d}}(x)=-\frac{\frac{5}{12} A\left(\frac{R_{0}}{x}\right)+\frac{x}{R_{0}}}{\left(A\left(\frac{R_{0}}{2 x}\right)^{2}+1\right)^{2}},
$$

where we assumed that $\epsilon_{\mathrm{d}}(x)$ is an odd function. The strain distribution $\epsilon_{\mathrm{r}}(x, R)$ for $R=R_{0}$ is given by Eqs. (2) and (5). As shown in Fig. 2(c), $\epsilon_{\mathrm{r}}\left(x, R_{0}\right)$ deviates from $x / R_{0}$ when $|x| / R_{0}$ increases, and exhibits its maximum value at $|x| / R_{0}=$ $0.341 \sqrt{A} . \epsilon_{\mathrm{r}}^{\max }(=0.183 \sqrt{A})$ with $A=0.0015$ is estimated at $0.71 \%$, the value of which is of the same order as the $\mathrm{x}$-ray diffraction measurement results for the ring-shaped crystals of $\mathrm{NbSe}_{3} .{ }^{21}$ For $|x| / R_{0} \gg 0.341 \sqrt{A}$, the strain $\epsilon_{\mathrm{r}}(x)$ slowly approaches zero as $|x| / R_{0}$ is increased.

We found that the distribution of the edge dislocations $n_{\mathrm{d}}(x)$ has two peaks at $x / R_{0}= \pm \tau / 2 \sim \pm 0.575 \sqrt{A}$, as shown in Fig. 2(c), which suggests that the dislocations gather on ring with radius $R=R_{0} \pm \tau / 2$. Here, we calculated $n_{\mathrm{d}}(x)=-\frac{2 \pi R_{0} a^{*}}{b} \frac{d}{d x} \epsilon_{\mathrm{d}}(x)$, where $a^{*}=a \sin \beta$ and $b$ are the lattice constants along the radial and circumference directions, respectively. The peaks indicate that elasticity is dominant when $\tau>T / R_{0}$ and plasticity is dominant when $\tau<T / R_{0}$. If edge dislocations gather on a circle in the ring-shaped crystals, the cores of the dislocations might overlap each other and form a cylindrical domain wall as anticipated in our previous paper, ${ }^{21}$ similar to the cylindrical vortices in the ring-shaped crystals of $\mathrm{TaSe}_{3} .{ }^{18}$ The cylindrical domain walls can relax the bending stress homogeneously conserving the global disclination. If the strain $\epsilon=\tau / 2$ is relaxed to zero by a cylindrical domain wall, one edge dislocation is induced for approximately every 45 lattices along the b axis $\left(N_{\mathrm{d}}=\frac{2 \pi R_{0}}{b} \frac{\tau}{2}\right)$. The value corresponds fairly well with the order of core size of the edge dislocations along the $\mathbf{b}$ axis $\left(\Lambda_{y}=a^{*} / \gamma\right.$, where $\gamma \sim 10^{-1}$ is the parameter representing anisotropy of the crystal structure ${ }^{20}$ ). Thus, the domain size $\tau$ is determined by the circumference length of the ring-shaped crystals. This must be the interplay between the global topology and the local crystal orders.

Furthermore, the cylindrical domain walls would be introduced periodically in thick ring-shaped crystals of $T / R_{0}>2 \tau$. 
(a)

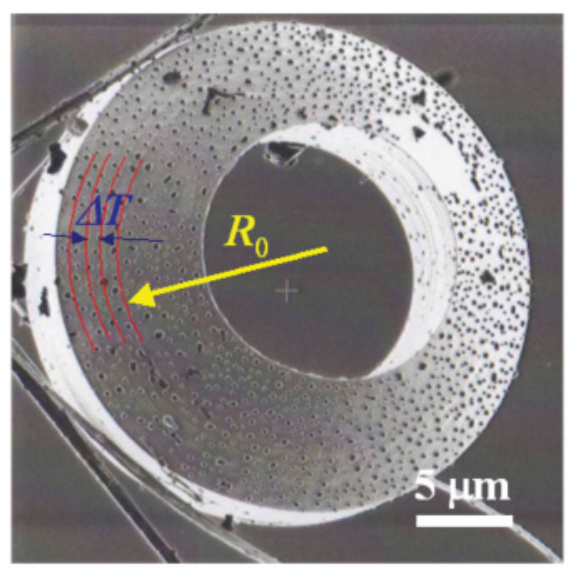

(b)

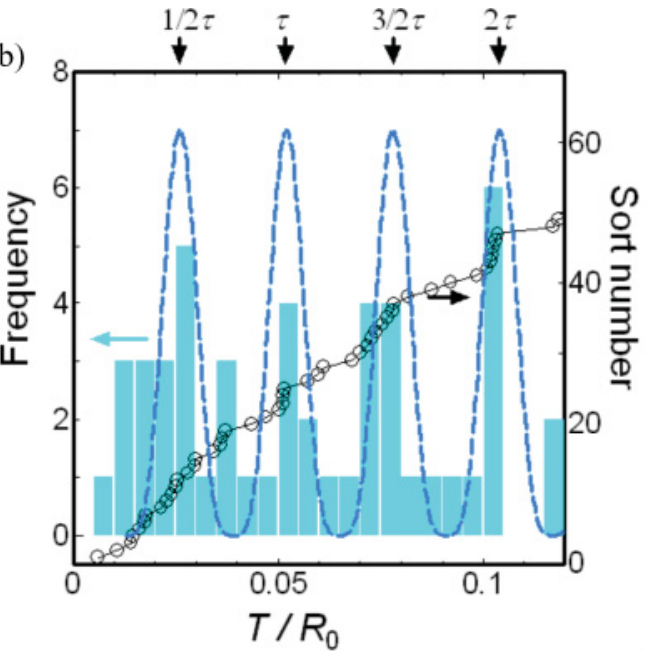

(c)

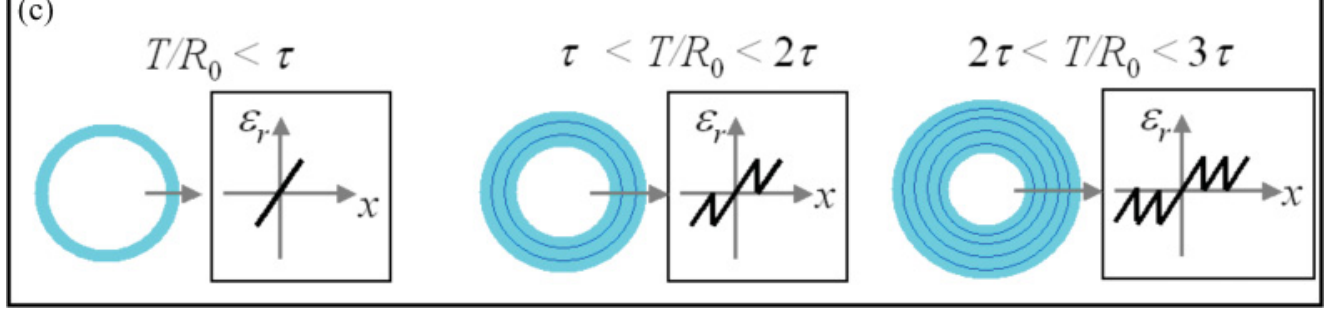

FIG. 3. (Color online) (a) SIM image of a thick ring-shaped crystal of TaSe $\mathrm{T}_{3}$. On the surface, the concentric lines with a period of $\Delta T \sim 0.8 \mu \mathrm{m}$ are observed (indicated by the red curves). $R_{0}=11 \mu \mathrm{m}$ and $\Delta T / R_{0} \sim 0.072$, which is in the order of $\tau$. On the lines, the selenium droplets are also observed. (b) Frequency distribution of randomly selected ring-shaped crystals as a function of $T / R_{0}$. The blue broken curve is a guide for the eyes showing the periodic peaks of the frequency distribution. The black open circles indicate the sort number of each ring-shaped crystal numbered in order starting with the smallest $T / R_{0}$ value, and show the periodic step structures. (c) Model of cylindrical domain walls in the ring-shaped crystal and strain distribution inside crystal.

As shown Fig. 3(a), we found the concentric rings on the surface of some thick ring-shaped crystals (indicated by red curves), and on which the selenium droplets congregated. The period $\left(\Delta T / R_{0}\right)$ of the rings is in the order of $\tau$. We also found the frequency (number) distribution of normalized thickness, $T / R_{0}$, of randomly selected ring-shaped crystals oscillating in the period of $\tau / 2$, as shown in the histogram of Fig. 3(b). The number of the rings of a value of $T / R_{0}$ would increase if the growing speed of thickness becomes slow at the thickness. Hence, the oscillation implies that the cylindrical domain walls induce periodically as a function of $T / R_{0}$. When $T / R_{0}$ becomes large, the growing speed of thickness $T$ for the ring-shaped crystals decreases due to an increase in the bending stress energy [Fig. 3(c), left panel], and if a cylindrical domain wall is introduced, as shown in Fig. 3(c) for $T / R_{0}>\tau$, the stress is relaxed, and then the growing speed recovers. These two results suggest that introducing the cylindrical domain walls is the main mechanism of relaxation for the bending strain in thick ring-shaped crystals. We note that for $T / R_{0}>\tau,\left(R_{\mathrm{f}}-R_{0}\right) / R_{0}$ would oscillate with a period of $\tau$ due to the introduction of the domain walls; however, the amplitude of the oscillation would be smaller than the experimental noise in Fig. 1(d). The defect density between the two domain walls should be small, the crystal structures would be clean as well as single crystals. These features of the crystals are consistent with the results of the previous experiments in which we have observed clear charge density waves and superconductivity in the ring-shaped crystals as well as single crystals. ${ }^{10-15,18}$

In summary, we demonstrated that the topology-change surgery gives us valuable information in crystals. By cutting off the ring-shaped crystals of $\mathrm{TaSe}_{3}$, we found a phenomenological relation between the radius of curvature after the topology change and thickness. From the relation, we extracted the crystal domain size $(\tau)$ of the ring-shaped crystals. These results strongly suggest that the cylindrical domain walls are induced in the ring-shaped crystals, due to the interplay between the closed-ring topology and the crystal structures. The topology-change surgery is a significant methodology to extract topological effects, and would be applicable to other topological materials, such as multiwall nanotubes ${ }^{27,28}$ and biomolecules. $^{5}$

We thank M. Oda, H. Orihara, M. Hayashi, Y. Nogami, K. Yamamoto, T. Shibayama, K. Yamaya, K. Inagaki, T. Toshima, and H. Okawa for their fruitful discussions and supports. This research was supported by the Center of Education and Research for Topological Science and Technology, Hokkaido University, and the Japan Society for the Promotion Science. 
${ }^{1}$ M. Dehn, Acta Math. 69, 135.

${ }^{2}$ G. Perelman, e-print arXiv:math/0303109v1.

${ }^{3}$ J. P. Luminet, J. R. Weeks, A. Riazuelo, R. Lehoucq, and J. P. Uzan, Nature 425, 593 (2003).

${ }^{4}$ G. E. Volovik, Trans. R. Soc. A 366, 2935 (2008).

${ }^{5}$ Y. Timsit and P. Várnai, PLoS ONE 5, e9326 (2010).

${ }^{6}$ S. Tanda, T. Tsuneta, A. Y. Okajima, K. Inagaki, K. Yamaya, and N. Hatakenaka, Nature 417, 397 (2002).

${ }^{7}$ T. Tsuneta and S. Tanda, J. Cryst. Growth 264, 223 (2004).

${ }^{8}$ S. Tanda, H. Kawamoto, M. Shiobara, Y. Okajima, and K. Yamaya, J. Phys. IV 9, 379 (1999).

${ }^{9}$ T. Matsuura, M. Yamanaka, N. Hatakenaka, T. Matsuyama, and S. Tanda, J. Cryst. Growth 297, 157 (2006).

${ }^{10}$ Y. Okajima, H. Kawamoto, M. Shiobara, K. Matsuda, S. Tanda, and K. Yamaya, Physica B 284, 1659 (2000).

${ }^{11}$ T. Tsuneta, S. Tanda, K. Inagaki, Y. Okajima, and K. Yamaya, Physica B 329-333, 1544 (2003).

${ }^{12}$ T. Matsuura, K. Inagaki, and S. Tanda, Phys. Rev. B 79, 014304 (2009).

${ }^{13}$ M. Tsubota, K. Inagaki, and S. Tanda, e-print arXiv:0906.5206.

${ }^{14}$ T. Matsuura, T. Tsuneta, K. Inagaki, and S. Tanda, Phys. Rev. B 73, 165118 (2006).

${ }^{15}$ K. Shimatake, Y. Toda, and S. Tanda, Phys. Rev. B 73, 153403 (2006).

${ }^{16}$ T. Nogawa and K. Nemoto, Phys. Rev. B 73, 184504 (2006).
${ }^{17}$ M. Hayashi, H. Ebisawa, and K. Kuboki, Phys. Rev. B 76, 014303 (2007).

${ }^{18}$ G. Kumagai, T. Matsuura, K. Ichimura, K. Yamaya, K. Inagaki, and S. Tanda, Phys. Rev. B 81, 184506 (2010).

${ }^{19}$ A. E. Romanov and V. I. Vladimirov, "Disclinations in crystralline solids," in Dilocations in Solids, edited by F. R. N. Nabarro (Elsevier Science Publishers B.V., Amsterdam, 1992), Vol. 9, Chap. 47.

${ }^{20}$ M. Hayashi, H. Ebisawa, and K. Kuboki, Europhys. Lett. 76, 264 (2006).

${ }^{21}$ T. Tsuneta, K. Yamamoto, N. Ikeda, Y. Nogami, T. Matsuura, and S. Tanda, Phys. Rev. B 82, 014105 (2010).

${ }^{22}$ E. Bjerkelund and A. Kjekshus, Acta Chem. Scand. 19, 701 (1966).

${ }^{23}$ T. Sambongi, M. Yamamoto, K. Tsutsumi, Y. Shiozaki, K. Yamaya, and Y. Abe, J. Phys. Soc. Jpn. 42, 1421 (1977).

${ }^{24}$ J. L. Hodeau, M. Marezio, C. Roucau, R. Ayroles, A. Meerschaut, J. Rouxel, and P. Monceau, J. Phys. C 11, 4117 (1978).

${ }^{25}$ T. Matsuura, S. Tanda, K. Asada, Y. Sakai, T. Tsuneta, K. Inagaki, and K. Yamaya, Physica B 329, 1550 (2003).

${ }^{26}$ L. D. Landau, L. P. Pitavskii, E. M. Lifshitz, and A. M. Kosevich, Theory of Elasticity, 3rd ed., Vol. 7 (Theoretical Physics) (Butterworth-Heinemann, United Kingdom, 1986).

${ }^{27}$ D. V. Kosynkin, A. L. Higginbotham, A. Sinitskii, J. R. Lomeda, A. Dimiev, B. K. Price, and J. M. Tour, Nature 458, 872 (2009).

${ }^{28}$ L. Jiao, L. Zhang, X. Wang, G. Diankov, and H. Dai, Nature 458 , 877 (2009). 